
allemande

51-1 | 2019

La République démocratique allemande dans l'espace public européen (1949-2018)

\title{
Aurélie CHONÉ, Isabelle HAJEK et Philippe HAMMAN (éd.), Guide des Humanités environnementales
}

Villeneuve d'Ascq, Presses universitaires du Septentrion, 2016

\section{Cécile Fries-Paiola}

\section{OpenEdition}

\section{Journals}

Édition électronique

URL : https://journals.openedition.org/allemagne/1648

DOI : $10.4000 /$ allemagne. 1648

ISSN : 2605-7913

Éditeur

Société d'études allemandes

Édition imprimée

Date de publication : 2 juillet 2019

Pagination : 265-267

ISSN : 0035-0974

Référence électronique

Cécile Fries-Paiola, « Aurélie choné, Isabelle hasek et Philippe hamman (éd.), Guide des Humanités environnementales », Revue d'Allemagne et des pays de langue allemande [En ligne], 51-1 | 2019, mis en ligne le 02 juillet 2019, consulté le 02 juin 2022. URL : http://journals.openedition.org/allemagne/1648 ; DOI : https://doi.org/10.4000/allemagne.1648 


\section{Italiques}

Aurélie Choné, Isabelle Hajek et Philippe Hamman (éd.), Guide des Humanités environnementales, Villeneuve d'Asca, Presses universitaires du Septentrion (coll. Environnement et société), 2016, 632 p.

Comme son titre l'indique, il s'agit, plutôt que d'un dictionnaire - dont il ne revendique ni l'exhaustivité des entrées, ni le format de notices -, d'un état des lieux et d'un croisement des principales approches contemporaines, portées par les Humanités au sens large, qui nous permettent de penser le rapport qu'entretiennent nos sociétés avec la nature. Remarquons d'emblée un parti pris intéressant de l'ouvrage qui mobilise en son cour le terme de «nature» - parfois aujourd'hui abandonné à tort par commodité conceptuelle - permettant ainsi de la "confirm[er] dans son épaisseur» (p. 18).

Tant sur la forme que sur la thématique, cet ouvrage développe des spécificités intéressantes. D'une part, ce guide prend la forme d'un ouvrage dense de 632 pages, regroupant 53 contributions - chapeautées par une introduction - et réparties selon deux grands axes de questionnement: "comment penser la nature?» et «comment vivre avec la nature?». Cette structure permet non seulement une lecture linéaire, mais également une lecture croisée, selon un enchaînement de différentes entrées choisies au gré des thématiques de recherche propres à chaque lecteur - ce qui permet ainsi de créer des passerelles fertiles entre la multiplicité des différentes approches thématiques ou disciplinaires. D'autre part, l'ambition thématique et théorique de l'ouvrage est claire et annoncée dès l'introduction: "constituer un support de référence centré sur la polysémie et l'épaisseur de l'idée de nature telle qu'elle a été développée dans les histoires culturelles européennes de la modernité, au cours des XIXe et XXe siècles; et montrer comment celle-ci, dans les défis qu'elle adresse aujourd'hui aux sociétés contemporaines, reconfigure les cadres de pensée, les disciplines et leurs objets de recherche pour produire de nouveaux champs de questionnements et de pratiques» (p. 15).

Ainsi, cet ouvrage s'inscrit dans un mouvement récent de sommes, qui prennent diverses formes et qui visent à appréhender la relation homme/nature dans une certaine globalité (historique ou thématique). Citons par exemple le Dictionnaire de la 
pensée écologique ${ }^{(1)}$, La pensée écologique ${ }^{(2)}$, ou encore Pour une sociologie de l'environnement: environnement, société et politique ${ }^{(3)}$. Cette filiation est d'ailleurs affirmée par les directeurs de publication de ce Guide des Humanités environnementales qui prennent soin, en introduction, de se positionner par rapport à nombre d'ouvrages qui pourraient apparaître comme voisins, en défendant la spécificité de leur approche, marquée notamment par une large pluridisciplinarité, des incursions dans des "ailleurs", et des dimensions historique et critique transversales à l'ouvrage. Et ce sont effectivement ces trois aspects qui font toute la richesse et la singularité de ce guide.

Dans un premier temps, il est intéressant de souligner la véritable pluridisciplinarité de cet ouvrage et la place centrale faite à l'ensemble des Humanités. Ainsi, l'approche juridique est bien représentée, et paraît aujourd'hui plus que pertinente pour comprendre comment nos sociétés appréhendent la nature, mais aussi pour pouvoir envisager des solutions à un engrenage institutionnel qui semble parfois dépasser nos volontés d'action individuelles face à une nature en danger. L'entrée "Écocide» en est une excellent exemple, cette notion s'imposant désormais de manière incontournable dans le débat public. Par ailleurs, l'approche esthétique, trop souvent délaissée dans l'appréhension de la relation homme/nature, est largement valorisée dans ce guide. Qu'il s'agisse d'entrées plus théoriques, telles que celles de l'«Esthétique de l'environnement», de l'"Esthétique de la nature», ou de l'«Écocritique», ou d'entrées plus thématiques autour de la «Forêt», de la «Montagne» et du «Paysage», toutes révèlent à quel point les arts, et la littérature en particulier, permettent de mieux comprendre la construction des catégories qui président à l'appréhension et la définition de la nature, influençant in fine nos manières d'être à la nature.

Dans un second temps, il paraît important de mettre en avant la particulière actualité de certaines thématiques abordées. Il en va notamment des cinq entrées relatives à l'animal et au vivant. La contribution portant sur la "protection juridique de l'animal» fait bien entendu sens par rapport aux préoccupations sociétales contemporaines, mais permet surtout de comprendre l'évolution et les enjeux du regard que nous portons sur cette portion du vivant, à laquelle nous avons des difficultés à nous assimiler. Tel un miroir, interroger notre définition de l'animalité, c'est en réalité interroger notre humanité et la façon de considérer ou non, de prendre soin ou non, de l'autre. Dès lors, ce guide prend toute son épaisseur, lorsqu'il nous invite à entrevoir que notre rapport à la nature ne pourra être compris que dans la mesure où nous parviendrons à entretenir un rapport conscient et bienveillant à ce qui constitue notre propre humanité. Des approches telles que celle de l' "Écopsychologie», dont est à l'origine le sociologue et historien américain Theodore Rozsak, qui met notamment en regard le désordre de nos paysages intérieurs et de nos paysages extérieurs, proposent des paradigmes pertinents pour cesser de dresser des barrières aussi péremptoires que destructrices, entre les dimensions humaines et naturelles.

Dans un troisième et dernier temps, pour terminer ce rapide tour d'horizon de cet ouvrage foisonnant, notons que les entrées spatiales, et particulièrement urbaines, ne sont pas en reste. Si l'un des enjeux d'un tel ouvrage, bien que non revendiqué, serait celui d'une certaine opérationnalité des résultats de recherche ici exposés, alors ce sont

1 Dominique Bourg, Aurélien PAPAuX (dir.), Dictionnaire de la pensée écologique, Paris, Presses universitaires de France, 2015.

2 Dominique BourG, Augustin FrAGNiÉRE (dir.), La pensée écologique, une anthologie, Paris, Presses universitaires de France, 2014.

3 Bernard KALAORA, Chloé Vlassopoulos, Pour une sociologie de l'environnement: environnement, société et politique, Seyssel, Champ Vallon, 2013. 
bien du côté des entrées de la "Ville naturante», de l'«Écologie urbaine», de l'«Urbanisme durable», des "Quartiers durables», ou encore de la "Nature en ville» que cette dimension se manifeste. Ces contributions, en regard d'approches plus théoriques et issues d'autres champs thématiques et disciplinaires, pourraient permettre à des acteurs de la ville et des territoires de s'interroger sur les moyens mis en œuvre pour tendre à un aménagement des espaces habités plus soucieux de la nature, comme des habitants.

Ainsi, cet ouvrage constitue avec certitude un apport riche et central pour appréhender dans une globalité thématique et disciplinaire le rapport homme/nature. Des ouvertures possibles apparaissent à la fin de la lecture de l'ouvrage, pour poursuivre cette vaste quête épistémologique, dont ce guide est l'initiateur. Le panel disciplinaire et thématique de l'ouvrage est large. D'autres approches pourraient venir compléter celles déjà présentes. L'économie et la démographie, ainsi que des entrées autour des pensées de la résilience, de la décroissance et des biens communs constituent également des pistes de réflexion possibles pour penser notre rapport à la nature.

En conclusion, cet ouvrage propose des contributions de qualité, largement éclairantes pour tous ceux qui ont à penser, quelle que soit leur discipline, le rapport que nous entretenons aujourd'hui avec ce que nous ne sommes parfois plus tout à fait certains de pouvoir appeler «nature». Le Guide des Humanités environnementales ouvre un chemin, qu'il est désormais plus que nécessaire de poursuivre: mettre en commun et faire dialoguer les recherches contemporaines issues de toutes les sciences humaines et sociales portant sur les relations "humain/non-humain» (4) - qu'il s'agisse de la nature, de l'environnement ou encore du milieu -, en vue, espérons-le, non seulement d'en construire une connaissance toujours plus aiguisée mais également de trouver des leviers d'action concrets pour faire face à la crise environnementale (mais aussi sociale, économique, etc.) dans laquelle nous sommes tous engagés pour les siècles à venir.

Cécile Fries-Paiola

\title{
Katrin JORDAN, Ausgestrahlt. Die mediale Debatte um "Tschernobyl" in der Bundesrepublik und in Frankreich. 1986/87, Göttingen, Wallstein Verlag, 2018, $424 p$.
}

\begin{abstract}
Historienne spécialisée dans les relations franco-allemandes du XXe siècle et l'histoire des médias, Katrin Jordan livre une version remaniée de la thèse de doctorat qu'elle a menée à l'Université Humboldt de Berlin et au Centre d'histoire contemporaine de Potsdam, tout en ayant bénéficié de séjours en France, notamment à l'Institut historique allemand de Paris. Soutenue en février 2018, cette recherche a été récompensée par le Prix allemand 2019 du jeune talent en histoire de la communication. Le sujet retient immédiatement l'attention: la catastrophe nucléaire de Tchernobyl en 1986 et ce que son traitement public et médiatique différent en France et en Allemagne donne à saisir de rapports contrastés au nucléaire civil, du point de vue des décideurs et exprimés à travers divers relais d'opinion, qui produisent eux aussi une empreinte.

Sur de tels enjeux, les faiblesses d'un modèle explicatif - qu'il soit économique, technique, de sécurité nationale ou autre - participent de la portée d'un autre et
\end{abstract}

4 Philippe Descola, Par-delà nature et culture, Paris, Gallimard (coll. Bibliothèque des sciences humaines), 2005. 